\title{
Four Poynting theorems
}

\author{
Paul Kinsler, Alberto Favaro, and Martin W. McCall \\ Blackett Laboratory, Imperial College, Prince Consort Road, London SW7 2AZ, United Kingdom.
}

(Dated: November 9, 2016)

\begin{abstract}
The Poynting vector is an invaluable tool for analysing electromagnetic problems. However, even a rigorous stress-energy tensor approach can still leave us with the question: is it best defined as $\vec{E} \times \vec{H}$ or as $\vec{D} \times \vec{B}$ ? Typical electromagnetic treatments provide yet another perspective: they regard $\vec{E} \times \vec{B}$ as the appropriate definition, because $\vec{E}$ and $\vec{B}$ are taken to be the fundamental electromagnetic fields. The astute reader will even notice the fourth possible combination of fields: i.e. $\vec{D} \times \vec{H}$. Faced with this diverse selection, we have decided to treat each possible flux vector on its merits, deriving its associated energy continuity equation but applying minimal restrictions to the allowed host media. We then discuss each form, and how it represents the response of the medium. Finally, we derive a propagation equation for each flux vector using a directional fields approach; a useful result which enables further interpretation of each flux and its interaction with the medium.
\end{abstract}

Published in Eur. J. Phys. 30, 983 (2009). ${ }^{1}$

This arXiv version has updates not present in the published version.

\section{INTRODUCTION}

The correct definition of electromagnetic flux has long been controversial, with the main competition being between the Abraham $\vec{E} \times \vec{H}[1,2]$ and Minkowski $\vec{D} \times \vec{B}$ [3] forms. Pfeifer et al. [4] gave an excellent discussion and historical review of the situation, with an analysis based on energy-momentum tensors. In contrast, the Poynting theorem [5] and Poynting vector $\vec{S}=\vec{E} \times \vec{H}$, in concert with the electromagnetic energy density, lead us to an energy continuity equation. This equation is easily interpreted when considering only fields in the vacuum or in nondispersive linear media [6]. Further alternatives exist where the Poynting vector is generalized to include extra terms and so generate other equally valid flux vectors and energy densities [7-11]; however all these were based on $\vec{E} \times \vec{H}$.

However, outside the context of energy-momentum tensor definitions, but nevertheless common in electromagnetic usage, is the $\vec{E} \times \vec{B}$ form [12, 13]. Curiously, comparison or contrast of the Abraham/ Minkowski forms with $\vec{E} \times \vec{B}$ is hard to find - e.g. [4] does not remark on the different origins of $\vec{E} \times \vec{B}$ at all. Further, even though (e.g.) both $\vec{E} \times \vec{H}$ and $\vec{E} \times \vec{B}$ appear in [14], this is outside the context of magnetic media, so that $\vec{H} \equiv \vec{B} / \mu_{0}$ in any case.

In this paper we address the question: if we construct electromagnetic flux vectors ("Poynting vectors") using the cross product of two fields, what do the results look like, and what might we apply them to? How should non-trivial polarization and magnetization effects be interpreted? Also, does the alternative $\vec{D} \times \vec{H}$ combination of field vectors give interesting results? We allow for reasonably general propagation media, with potentially dispersive and nonlinear properties affecting both electric and magnetic fields. Such complications mean that we do not derive continuity equations with a perfectly balanced flux and energy density, but they also include extra "residual" terms: e.g. the standard $\vec{E} \times \vec{B}$ derivation produces a residual "work done" term $\vec{E} \cdot \vec{J}$, where $\vec{J}$ is the total current $[13,15]$. We show that

\footnotetext{
*Electronic address: Dr.Paul.Kinsler@physics.org

1 This is an author-created, un-copyedited version of an article accepted for publication in the European Journal of Physics. IOP Publishing Ltd is not responsible for any errors or omissions in this version of the manuscript or any version derived from it. The definitive publisher-authenticated version is available online at doi:10.1088/0143-0807/30/5/007.
}

in each case the residuals contain either temporal or spatial derivatives, and that they can be interpreted in terms of currents; we further show how they affect the propagation of the flux vector.

Since typical response models for a propagation medium, especially in the nonlinear case, are likely to be non-covariant, we tie our description to the medium rest frame without further loss of generality. Although this is a restriction we might prefer to avoid, as done for negative refraction by McCall [16], it usually has few consequences. Further, since our interest is primarily on propagating fields in nontrivial media, we leave consideration of interfaces, as well as surface and volume integrals, for later work; likewise we do not address the uses of the vector potential $\vec{A}$ (or its dual $[17,18]$ ) in this context.

The paper is organized as follows: in section II we introduce Maxwell's equations in the forms in which they are used in this paper, in section III we briefly remark on continuity equations, and in section IV we define our four electromagnetic flux vectors and their associated continuity equations. In section $\mathrm{V}$ we show how the residual terms modify the underlying propagation of the flux vectors, and we discuss all the results in section VI. Finally, in section VII we present our conclusions.

Our presentation is pedagogic in that most of the discussion is at the level of an undergraduate course in electromagnetism. The material presented could form extension work at the point when Poynting's theorem is introduced. Those aspects concerning the propagation of the flux vector will be of most interest to specialists.

\section{MAXWELL'S EQUATIONS}

Maxwell's equations for the electric field $\vec{E}$ and magnetic field $\vec{B}$ in a medium are

$$
\begin{aligned}
\nabla \cdot \vec{E} & =\frac{1}{\varepsilon_{0}} \rho_{b}+\frac{1}{\varepsilon_{0}} \rho_{f}=\frac{1}{\varepsilon_{0}} \rho \\
\nabla \cdot \vec{B} & =0 \\
\nabla \times \vec{E} & =-\partial_{t} \vec{B} \\
\nabla \times \vec{B} & =\mu_{0} \vec{J}_{b}+\mu_{0} \vec{J}_{f}+\mu_{0} \varepsilon_{0} \partial_{t} \vec{E},
\end{aligned}
$$

where $\left(\rho_{b}, \vec{J}_{b}\right)$ and $\left(\rho_{f}, \vec{J}_{f}\right)$ are respectively the bound and free (charge, current) densities. As an alternative, we can define an elec- 
tric polarization $\vec{P}$ and magnetization $\vec{M}$, and

$$
\begin{aligned}
\vec{J}_{b} & =\vec{J}_{P}+\vec{J}_{M} \quad=\partial_{t} \vec{P}+\nabla \times \vec{M} \\
\rho_{b} & =-\nabla \cdot \vec{P} \\
\vec{D} & =\varepsilon_{0} \vec{E}+\vec{P} \\
\vec{H} & =\frac{1}{\mu_{0}} \vec{B}-\vec{M} .
\end{aligned}
$$

These allow us to rewrite Maxwell's equations as

$$
\begin{aligned}
\nabla \cdot \vec{D} & =\rho_{f} \\
\nabla \cdot \vec{B} & =0 \\
\nabla \times \vec{E} & =-\mu_{0} \partial_{t}(\vec{H}+\vec{M}) \\
\nabla \times \vec{H} & =\vec{J}_{f}+\partial_{t} \vec{D} \quad=\vec{J}_{f}+\partial_{t}\left(\varepsilon_{0} \vec{E}+\vec{P}\right) .
\end{aligned}
$$

We can even rewrite eqn. (11) in the unconventional form

$$
\begin{aligned}
\nabla \times \vec{D} & =-\varepsilon_{0} \mu_{0} \partial_{t}(\vec{H}+\vec{M})+\nabla \times \vec{P} \\
& =-\varepsilon_{0} \mu_{0} \partial_{t} \vec{H}-\varepsilon_{0} \mu_{0} \vec{K}_{b},
\end{aligned}
$$

where we have defined

$$
\begin{aligned}
& \vec{K}_{b}=\vec{K}_{P}+\vec{K}_{M} \quad=-\frac{1}{\varepsilon_{0} \mu_{0}} \nabla \times \vec{P}+\partial_{t} \vec{M}, \\
& \sigma_{b}=-\nabla \cdot \vec{M} .
\end{aligned}
$$

This $\vec{K}_{b}$ appears in the same place as a monopole current would if such were allowed; $\sigma_{b}$ is the bound magnetic pole density. Note that $\vec{K}_{b}$ and $\sigma_{b}$ are merely a way of representing the (local) material response; we are not claiming that some process actually generates true magnetic monopoles inside the material $[14]^{2}$. Strictly speaking, this is also true of the bound electric charge and its currents - they are a mechanism used solely to represent the behaviour of the medium. Further, and just as for the ficticious bound electric charge density, the ficticious bound monopole density necessarily integrates to zero over all space. Thus the material response could, in principle, be re-represented as magnetic dipoles instead of monopoles. Note that in using this effective monopole current, we are not going as far as Carpenter [19], who posits a complementary universe dominated by magnetic monopoles and with no charge in order to clarify some conceptual difficulties. If we were to include free magnetic monopoles and free magnetic monopole currents, then the continuity equations given below would exhibit a great deal more symmetry on exchange of electric and magnetic effects.

At this point is is worth noting that the equations above represent the effect of electric polarization in one of two ways: either as a current of bound charges (i.e. $\vec{J}_{P}=\partial_{t} \vec{P}$ ), or as a result of bound monopole current loops (i.e. $\vec{K}_{P}=\nabla \times \vec{P}$ ). They also represent the effect of magnetization similarly: either as a current of bound monoples (i.e. $\vec{K}_{M}=\partial_{t} \vec{M}$ ), or as a result of bound electric current loops (i.e. $\vec{J}_{M}=\nabla \times \vec{M}$ ). Our aversion to free magnetic monopoles, and the widespread acceptance of free electric charges may bias many readers toward an electric current picture involving $\vec{J}_{P}$ and $\vec{J}_{M}$ - but since these comprise bound charges which are merely a convenient fiction, there is no physical reason not to consider using bound monopoles, or even a mix of the two, if we have sufficient reason. Indeed, if

\footnotetext{
${ }^{2}$ Chapter 9 , section 3
}

we (microscopically) model the magnetization as arising from some field-induced or environmental distortion of a unit cell or molecule, there is little a priori reason not to model the magnetization as induced magnetic dipoles - we need not take the extra step of assuming the dipoles arise from some induced current loop.

\section{CONTINUITY EQUATIONS}

One of the major uses of flux (Poynting) vectors is in energy continuity equations, where we can examine the balance between flux and local storage of the energy in a medium. In electromagnetism, the flux vector that is usually chosen is the Abraham form of the Poynting vector $\vec{E} \times \vec{H}$, although in some contexts the Minkowski form $\vec{D} \times \vec{B}$ is chosen. Some authors prefer an $\vec{E} \times \vec{B}$ form for the Poynting vector (e.g. the recent [15]); but only in media with a magnetic response does this differ from the Abraham form in anything but scaling.

Our starting point is just a cross-product of two selected fields, one electric $(\vec{E}$ or $\vec{D})$ and one magnetic $(\vec{H}$ or $\vec{B})$. In concert with Maxwell's equations, such cross-products result in continuity equations of the form

$$
\nabla \cdot \vec{S}=\partial_{t} \mathcal{U}+\delta
$$

Here $\vec{S}$ is an energy flux based on our chosen pair of field vectors. The energy density $\mathcal{U}$ is a function of the fields used to construct the energy flux vector, and will in general contain all the terms that can be expressed as a simple time derivative of some function. The remaining term $\delta$ is some residual contribution. One way of avoiding these residual terms is to use an energy flux vector of the Umov form, i.e. as an energy density multiplied by a velocity vector, and not the traditional cross-product of fields (see e.g. [20]). In the Umov approach, any terms that are not part of the defined energy density are attributed to the behaviour of the energy flux.

We might now assume that the fields and medium are in some nearly steady state, where the time dependence of $\mathcal{U}$ is either zero, or its rapid oscillations average to zero; but that the residual terms remain significant. Here, any change in energy flow $\vec{S}$ is balanced by the residual terms $\delta$ which would most simply be a rate of energy removal or supply. However, it is perfectly possible for there to be more complicated responses, with a dependence on either time or space; e.g. an in-effect temporary storage of energy giving rise to an oscilliatory behaviour. This is treated more rigorously in section $\mathrm{V}$.

\section{FLUX VECTORS}

Here we will use the different forms of Maxwell's equations given above to generate four different electromagnetic energy continuity equations. The way we generate these continuity equations is straightforward: we take our chosen flux vector as defined by a cross product of an electric field and a magnetic field and take the divergence, using the standard vector identity

$$
\nabla \cdot(\vec{X} \times \vec{Y})=-\vec{X} \cdot(\nabla \times \vec{Y})+\vec{Y} \cdot(\nabla \times \vec{X}) .
$$

Then, by substituting in appropriate Maxwell's equations into the RHS to substitute for the curl terms, we generate continuity equations of the general form given in eqn. (17). With this style of derivation, there are some subtleties regarding the role of total and self-field contributions, as has been discussed by Campoz and Jimènez [21]. 
Lastly, if we so wish, we might also apply the modifications to our chosen $\vec{S}_{X Y}=\vec{X} \times \vec{Y}$ flux vector that have been applied to the (bare) Abraham $\vec{E} \times \vec{H}$ form [7-11] - e.g. adding terms of zero divergence to $\vec{S}_{X Y}$.

\section{A. Abraham $\vec{E} \times \vec{H}$}

This Abraham form of electromagnetic flux (Poynting) vector is the most widely used of all, consisting of the fields $\vec{E}$ and $\vec{H}$. Inserting $\vec{E} \times \vec{H}$ into the identity eq. (18), and using eqns. (3) and (12) gives us a continuity equation, i.e.

$$
\begin{aligned}
\nabla \cdot(\vec{E} \times \vec{H}) & =-\vec{E} \cdot(\nabla \times \vec{H})+\vec{H} \cdot(\nabla \times \vec{E}) \\
& =-\vec{E} \cdot \partial_{t} \vec{D}-\vec{E} \cdot \vec{J}_{f}-\vec{H} \cdot \partial_{t} \vec{B} .
\end{aligned}
$$

This is in itself a widely used expression, but we proceed further to get

$$
\begin{aligned}
\nabla \cdot(\vec{E} \times \vec{H})= & -\vec{E} \cdot\left(\vec{J}_{f}+\varepsilon_{0} \partial_{t} \vec{E}+\partial_{t} \vec{P}\right)-\vec{H} \cdot \mu_{0}\left(\partial_{t} \vec{H}+\partial_{t} \vec{M}\right) \\
= & -\frac{1}{2} \partial_{t}\left(\varepsilon_{0} \vec{E} \cdot \vec{E}+\mu_{0} \vec{H} \cdot \vec{H}\right) \\
& \quad-\vec{E} \cdot \vec{J}_{f}-\vec{E} \cdot \partial_{t} \vec{P}-\mu_{0} \vec{H} \cdot \partial_{t} \vec{M} \\
\nabla \cdot \vec{S}_{E H}= & -\partial_{t} U_{E H}-\vec{E} \cdot \vec{J}_{f}-\vec{E} \cdot \partial_{t} \vec{P}-\mu_{0} \vec{H} \cdot \partial_{t} \vec{M}
\end{aligned}
$$

where $\vec{S}_{E H}=\vec{E} \times \vec{H}$ and $2 \mathcal{U}_{E H}=\varepsilon_{0} \vec{E} \cdot \vec{E}+\mu_{0} \vec{H} \cdot \vec{H}$.

Because we chose the fields $\vec{E}$ and $\vec{H}$ to generate our flux vector, we necessarily find that both of these local residual excitations depend only on the temporal response of the medium (i.e. they are dispersive). This means we can replace $\partial_{t} \vec{P}$ with the ordinary electric current $\vec{J}_{P}$, and $\partial_{t} \vec{M}$ with a monopole current contribution $\vec{K}_{M}$ :

$$
\nabla \cdot \vec{S}_{E H}=-\partial_{t} u_{E H}-\vec{E} \cdot \vec{J}_{f}-\vec{E} \cdot \vec{J}_{P}-\mu_{0} \vec{H} \cdot \vec{K}_{M} .
$$

We cannot somehow introduce $\vec{J}_{M}=\nabla \times \vec{M}$ here without making assumptions: $\vec{J}_{M}$ because that relies on a pre-existing magnetization that varies in space.

In optics, researchers work from a starting point that is primarily concerned with dispersion; even if that dispersion has usually been purely dielectric in origin. It is natural, therefore, for the optics community to prefer the $\vec{E} \times \vec{H}$ form, because it treats material polarization and magnetization in a purely temporal manner - although we see here that we are then forced to represent any magnetic response by bound monopoles. In the introduction we suggested that the $\vec{E}$ and $\vec{H}$ fields could be regarded as the bare fields - and it is this optics inspired temporally-centric view in which this is true. Another nice feature of this choice is that the LHS is a purely spatial derivative (being a divergence $\nabla \cdot$ ), whereas on the RHS both the non- $\vec{J}_{f}$ terms (i.e. energy density term and residuals) are temporal derivatives.

\section{Instantaneous responses}

For the case of a medium with an instantaneous scalar response, we have $\vec{P}=\chi_{P} \vec{E}$, so that $\vec{E} \cdot \partial_{t} \vec{P} \equiv\left(\partial_{t} \vec{E} \cdot \vec{P}\right) / 2$, and so the polarization $\vec{P}$ can be incorporated into the energy density; the same can be done for the magnetization $\vec{M}$. Hence we have

$$
\begin{aligned}
\nabla \cdot(\vec{E} \times \vec{H})=- & \frac{1}{2} \partial_{t}\left[\varepsilon_{0}\left(1+\chi_{P}\right) \vec{E} \cdot \vec{E}+\mu_{0}\left(1+\chi_{M}\right) \vec{H} \cdot \vec{H}\right] \\
& -\vec{E} \cdot \vec{J}_{f} \\
= & -\frac{1}{2} \partial_{t}[\vec{E} \cdot \vec{D}+\vec{H} \cdot \vec{B}]-\vec{E} \cdot \vec{J}_{f}
\end{aligned}
$$

A consequence of this is that we can move any instantaneous linear part of the medium response into the energy density, leaving only the dispersive contributions to remain as residual terms. In practical terms, we can replace $\varepsilon_{0}$ and $\mu_{0}$ in eqn. (23) and associated definitions by instantaneous-response parameters $\varepsilon_{i}=\varepsilon_{0}\left(1+\chi_{P}\right)$ and $\mu_{i}=\mu_{0}\left(1+\chi_{M}\right)$, then only the non-instantaneous or nonlinear responses will remain in $\vec{P}$ and $\vec{M}$.

\section{B. Electric current: $\vec{E} \times \vec{B}$}

This definition uses the electric field $\vec{E}$ and the magnetic field $\vec{B}$ (see e.g. $[12,13]$ ), and has been recently favoured by some authors for use in complex media [15, 22-24]. However, one instance [23] has suffered significant (although initially disputed) comment [2527] as to its validity in certain situations. Leaving aside this dispute, we can still use the vector identity above to get a continuity equation; this will still be physically valid, even if using it in complex media may mislead the unwary.

To reiterate our earlier point - rather than attempting to justify a particular choice of flux vector with respect to some external criteria, we simply set up the definition(s), and determine what can be done on that basis. The continuity equation for $\vec{E} \times \vec{B}$ is based on eqns. (3) and (4), and is

$$
\begin{aligned}
\nabla \cdot(\vec{E} \times \vec{B}) & =-\vec{E} \cdot(\nabla \times \vec{B})+\vec{B} \cdot(\nabla \times \vec{E}) \\
& =-\vec{E} \cdot\left(\mu_{0} \vec{J}+\mu_{0} \varepsilon_{0} \partial_{t} \vec{E}\right)-\vec{B} \cdot \partial_{t} \vec{B} \\
& =-\frac{\mu_{0}}{2} \partial_{t}\left(\varepsilon_{0} \vec{E} \cdot \vec{E}+\frac{1}{\mu_{0}} \vec{B} \cdot \vec{B}\right)-\mu_{0} \vec{E} \cdot \vec{J} \\
\nabla \cdot \vec{S}_{E B} & =-\partial_{t} \mathcal{U}_{E B}-\vec{E} \cdot \vec{J},
\end{aligned}
$$

where of course $\vec{J}=\vec{J}_{f}+\partial_{t} \vec{P}+\nabla \times \vec{M}$; this is just the total current density; also $\mu_{0} \vec{S}_{E B}=\vec{E} \times \vec{B}$ and $2 \mathcal{U}_{E B}=\varepsilon_{0} \vec{E} \cdot \vec{E}+\vec{B} \cdot \vec{B} / \mu_{0}$.

The simple representation of the residual terms above (i.e. as $\vec{E} \cdot \vec{J}$ ) is presumably the underlying reason why $\vec{E} \times \vec{B}$ is preferred by many authors: the material response is represented in terms of an electric current, and $\vec{E} \cdot \vec{J}$ can be interpreted simply as the work done on charges. However, from a more general point of view, $\vec{E} \times \vec{B}$ is not the "correct" form of the Poynting vector, it just is one of many, each of which may (or may not) be more convenient in a particular situation. Note that here only the polarization residual excitation depends on the temporal response (i.e. dispersion); the magnetic part is purely spatial: both forms combine to generate a bound electric current. This is obviously an advantage to those who prefer to think only in terms of electric charges, whether real or ficticious; certainly $\vec{E} \times \vec{B}$ is the obvious choice for any microscopic model involving real charges (and no real monoples). However, the time response (dispersion) of the magnetization is no longer explicit, as it was for $\vec{E} \times \vec{H}$, but has become hidden inside the bound magnetization current $\vec{J}_{M}$.

In the previous subsection, we suggested that the $\vec{E}$ and $\vec{H}$ fields could be regarded as the bare fields. This is a point of view often taken in optics, and is consistent with the $\vec{E} \times \vec{H}$ choice of Poynting vector. However, choosing $\vec{E} \times \vec{B}$ gives us an alternative electric 
charge (or current) centric view, in which case it is the $\vec{E}$ and $\vec{B}$ fields which are regarded as the bare fields, indeed they are already widely regarded as the fundamental electromagnetic fields $[12]^{3}$ and $[13]^{4}$.

\section{Instantaneous and pointlike responses}

In the case of instantaneous polarization response and pointlike magnetic response, we find that eqn. (27) can be directly reduced to the same result as in eqn. (26), but scaled by $\mu_{0}$, since $\vec{H}$ has been replaced by $\vec{B}$ in the flux vector. A consequence of this is that we can move such components of the medium response into the energy density, leaving only the dispersive polarization and spatial magnetic parts as residual terms.

\section{Magnetic current: $\vec{D} \times \vec{H}$}

This alternate, and little (or never) used definition comprises the magnetic induction field $\vec{H}$ and the displacement field $\vec{D}$ as its basic components. Note that the historical review of Buchwald [28] makes some discouraging remarks as regards choosing $\vec{D}$ and $\vec{H}$ as fundamental fields at the end of chapter 2 (p.18), but makes no reference to a flux generated from them. Alternatively, the projection approach taken to pulse propagation by Kolesik et al. $[29,30]$ relied on the $\vec{D}$ and $\vec{H}$ fields, although they did not consider media with a magnetic response. The $\vec{D} \times \vec{H}$ continuity equation is based on eqns. (12), (14), and is

$$
\begin{aligned}
\nabla \cdot(\vec{D} \times \vec{H})= & -\vec{D} \cdot(\nabla \times \vec{H})+\vec{H} \cdot(\nabla \times \vec{D}) \\
=- & \vec{D} \cdot\left(\vec{J}_{f}+\partial_{t} \vec{D}\right) \\
& -\vec{H} \cdot\left(\varepsilon_{0} \mu_{0} \partial_{t} \vec{H}+\varepsilon_{0} \mu_{0} \partial_{t} \vec{M}-\nabla \times \vec{P}\right) \\
= & -\frac{\varepsilon_{0}}{2} \partial_{t}\left(\frac{1}{\varepsilon_{0}} \vec{D} \cdot \vec{D}+\mu_{0} \vec{H} \cdot \vec{H}\right) \\
& \quad-\vec{D} \cdot \vec{J}_{f}-\varepsilon_{0} \mu_{0} \vec{H} \cdot \partial_{t} \vec{M}+\vec{H} \cdot \nabla \times \vec{P} \\
\nabla \cdot \vec{S}_{D H}= & -\partial_{t} \mathcal{U}_{D H}-\frac{1}{\varepsilon_{0}} \vec{D} \cdot \vec{J}_{f}-\mu_{0} \vec{H} \cdot \partial_{t} \vec{M}+\frac{1}{\varepsilon_{0}} \vec{H} \cdot \nabla \times \vec{P}
\end{aligned}
$$

where $\varepsilon_{0} \vec{S}_{D H}=\vec{D} \times \vec{H}$ and $2 \mathcal{U}_{D H}=\vec{D} \cdot \vec{D} / \varepsilon_{0}+\mu_{0} \vec{H} \cdot \vec{H}$. Note that only the magnetization residual excitation depends on the temporal response (i.e. dispersion); the polarization part is purely spatial; as a result the material response can be encoded solely by means of the bound monopole current $\vec{K}_{b}$ defined in eqn. (15):

$$
\nabla \cdot \vec{S}_{D H}=-\partial_{t} \mathcal{U}_{D H}-\frac{1}{\varepsilon_{0}} \vec{D} \cdot \vec{J}_{f}-\mu_{0} \vec{H} \cdot \vec{K}_{b}
$$

although the free current part still mimics the $\vec{E} \cdot \vec{J}$ form.

This $\vec{D} \times \vec{H}$ form, therefore, is the natural complement to the electric current based $\vec{E} \times \vec{B}$ form, because the material response in both is completely encoded by means of a current: but one is a magnetic monople current $\vec{K}_{b}$, and one an ordinary electric current $\vec{J}_{b}$. It is probably unsurprising, therefore, that $\vec{D} \times \vec{H}$ has been neglected,

\footnotetext{
${ }^{3}$ Chapter 27, section 3

${ }^{4}$ Chapter 1 , section 1
}

because it treats material polarization and magnetization purely as monopole currents - even though these are fictitious monopoles, bound in dipole pairs, introduced solely to represent (model) the material response. Indeed, the description here is a purely continuum one, and the use of a monopole current is not contingent on the existence of fundamental particles carrying magnetic charge.

The $\vec{E} \times \vec{H}$ Poyting vector gave us a time-centric viewpoint, and was compatible with regarding $\vec{E}$ and $\vec{H}$ as the bare fields, similarly the electric-current centred viewpoint of $\vec{E} \times \vec{B}$ led us to insist that $\vec{E}$ and $\vec{B}$ are the bare fields. Here we have seen that a monopole-current centred viewpoint might encourage the idea that $\vec{D}$ and $\vec{H}$ are the bare electromagnetic fields!

\section{Pointlike and instantaneous responses}

In the case of pointlike polarization response and instantaneous magnetic response, we find that eqn. (31) can be directly reduced to the same result as in eqn. (26), but scaled by $\varepsilon_{0}$. A consequence of this is that we can move such components of the medium response into the energy density, leaving only the dispersive magnetic and spatial polarization parts as residual terms.

\section{Minkowski $\vec{D} \times \vec{B}$}

This uses the usual definition of the Minkowski Poynting vector, which contains the displacement and magnetic fields $\vec{D}$ and $\vec{B}$. Its continuity equation is based on eqns. (4) and (14), along with eqns. (5) and (7), and is

$$
\begin{aligned}
\nabla \cdot(\vec{D} \times \vec{B})= & -\vec{D} \cdot(\nabla \times \vec{B})+\vec{B} \cdot(\nabla \times \vec{D}) \\
=- & \vec{D} \cdot \mu_{0}\left(\vec{J}_{f}+\nabla \times \vec{M}+\partial_{t} \vec{D}\right) \\
& +\vec{B} \cdot\left(-\varepsilon_{0} \partial_{t} \vec{B}+\nabla \times \vec{P}\right) \\
= & -\frac{\mu_{0} \varepsilon_{0}}{2} \partial_{t}\left(\frac{1}{\varepsilon_{0}} \vec{D} \cdot \vec{D}+\frac{1}{\mu_{0}} \vec{B} \cdot \vec{B}\right) \\
& \quad-\mu_{0} \vec{D} \cdot\left(\vec{J}_{f}+\nabla \times \vec{M}\right)+\vec{B} \cdot \nabla \times \vec{P} \\
\nabla \cdot \vec{S}_{D B}= & -\partial_{t} \mathcal{U}_{D B}-\frac{1}{\varepsilon_{0}} \vec{D} \cdot\left(\overrightarrow{J_{f}}+\nabla \times \vec{M}\right)+\frac{1}{\varepsilon_{0} \mu_{0}} \vec{B} \cdot \nabla \times \vec{P}
\end{aligned}
$$

where $\varepsilon_{0} \mu_{0} \vec{S}_{D B}=\vec{D} \times \vec{B}$ and $2 \mathcal{U}_{D B}=\vec{D} \cdot \vec{D} / \varepsilon_{0}+\vec{B} \cdot \vec{B} / \mu_{0}$.

Note that neither residual excitation depends on the temporal response (i.e. dispersion); the polarization and magnetization parts are purely spatial. This means that we might replace $\nabla \times \vec{M}$ with the electric current $\vec{J}_{M}$, and $\nabla \times \vec{P}$ with the monopole current $\vec{K}_{P} / \varepsilon_{0} \mu_{0}$ :

$$
\nabla \cdot \vec{S}_{D B}=-\partial_{t} \mathcal{U}_{D B}-\frac{1}{\varepsilon_{0}} \vec{D} \cdot\left(\vec{J}_{f}+\vec{J}_{M}\right)-\vec{B} \cdot \vec{K}_{P}
$$

This form, therefore, treats material polarization and magnetization in a purely spatial manner; as such it promotes a picture wherein it is the $\vec{D}$ and $\vec{B}$ fields which look like the bare fields.

\section{Pointlike responses}

In the case of pointlike magnetic and polarization responses, we find that eqn. (36) can be directly reduced to the same result as in eqn. 
(26), but scaled by $\varepsilon_{0} \mu_{0}$, since $\vec{E}$ and $\vec{H}$ have been replaced by $\vec{D}$ and $\vec{B}$. A consequence of this is that we can move such components of the medium response into the energy density, leaving simplified residual terms.

\section{PROPAGATION OF FLUX}

We can now show how these residual terms affect a propagating wave, by deriving propagation equations for the Poynting vectors themselves. To do this we use the concept of directional electromagnetic fields [31-33], and as a result do not need to resort to restrictive approximations, such as e.g. assuming plane wave or harmonic fields. Notably, we will not need to resort to ad-hoc time averaging of fast-oscillating terms, as done in this kind of context by e.g. Markel and others [24, 34]; neither do we need to introduce pulse envelopes, co-moving frames, or make smoothness assumptions [35-38].

First we note that each of our electromagnetic continuity eqns. (22), (29), (33), (38), has the general form

$$
-c \nabla \cdot(\vec{X} \times \vec{Y})=\frac{1}{2} \partial_{t}[\vec{X} \cdot \vec{X}+\vec{Y} \cdot \vec{Y}]+\vec{R} .
$$

Now assume transverse fields propagating in the direction given by a unit vector $\vec{u}$, so that $\vec{X} \cdot \vec{u}=\vec{Y} \cdot \vec{u}=0$. This means we can construct directional fields $[31,32]$ by defining

$$
\vec{G}^{ \pm}=\vec{X} \mp \vec{u} \times \vec{Y} ;
$$

where e.g. for plane polarized fields we might use $X_{x}=\sqrt{\varepsilon_{0}} E_{x}=$ $D_{x} / \sqrt{\varepsilon_{0}}$ and $Y_{y}=\sqrt{\mu_{0}} H_{y}=B_{y} / \sqrt{\mu_{0}}$; however note that we need not be restricted to scaling by the vacuum values of $\varepsilon_{0}$ and $\mu_{0}$. Thus since $\vec{X} \times \vec{Y}=\vec{u}\left(\left|\vec{G}^{+}\right|^{2}-\left|\vec{G}^{-}\right|^{2}\right) / 4$, we can write

$$
-c \nabla \cdot \vec{u}\left[\left|\vec{G}^{+}\right|^{2}-\left|\vec{G}^{-}\right|^{2}\right]=\frac{1}{4} \partial_{t}\left[\left|\vec{G}^{+}\right|^{2}+\left|\vec{G}^{-}\right|^{2}\right]+\vec{R},
$$

which is easily rearranged to

$$
\left[\partial_{t}-c \nabla \cdot \vec{u}\right]\left|\vec{G}^{+}\right|^{2}+\left[\partial_{t}+c \nabla \cdot \vec{u}\right]\left|\vec{G}^{-}\right|^{2}=-4 \vec{R} .
$$

This contains two counter-propagating components, each evolved by its own wave operator $\partial_{t} \mp c \nabla \cdot \vec{u}$, with the residual terms remaining on the RHS. If we are only interested in unidirectional propagation we can set $\vec{G}^{-}=0$, and with $\vec{u} \| \hat{z}$, we get

$$
\left[\partial_{t}-c \partial_{z}\right]\left|\vec{G}^{+}\right|^{2}=-4 \vec{R}
$$

This is a simple first order wave equation for the intensity, and we can easily see that loss-like residual terms $\vec{R}$ will cause that intensity to diminish; similarly those dependent on the past can cause dispersion. However, in the same way as with standard directional fields [31] or factorization approaches $[39,40]$, we require that the residual terms only have a small effect over the scale of one wavelength in order for $\vec{G}^{-}$to stay negligible [41-43].

Note that a considerable amount of detail is hidden in the total residual term $\vec{R}$; the various residual components are summarized in table I for each combination of field vectors. If we wanted to solve eqn. (45) (or perhaps even (44)), we would need to rewrite those residual components in terms of $\vec{G}^{ \pm}$; the approximation $\vec{G}^{-}=0$ helps in this respect since it allows the field choice $\vec{X}$ to be expressed in terms of $\vec{Y}$. In any case, we can see that the components of $\vec{R}$ will act as source terms that drive and modify the otherwise simple linear wave propagation.

TABLE I: Summary of flux vectors and their corresponding energy densities, along with the residual terms and their corresponding bound currents.

\begin{tabular}{lcccc}
\hline Flux & Energy density & Temporal & Spatial & Currents \\
\hline$\vec{E} \times \vec{H}$ & $\varepsilon_{0} \vec{E} \cdot \vec{E}+\mu_{0} \vec{H} \cdot \vec{H}$ & $\vec{E} \cdot \partial_{t} \vec{P}+\vec{H} \cdot \partial_{t} \vec{M}$ & 0 & $\vec{J}_{P}, \vec{K}_{M}$ \\
$\vec{E} \times \vec{B}$ & $\varepsilon_{0} \vec{E} \cdot \vec{E}+\mu_{0}^{-1} \vec{B} \cdot \vec{B}$ & $\vec{E} \cdot \partial_{t} \vec{P}$ & $+\vec{E} \cdot \nabla \times \vec{M}$ & $\vec{J}_{b}$ \\
$\vec{D} \times \vec{H}$ & $\varepsilon_{0}^{-1} \vec{D} \cdot \vec{D}+\mu_{0} \vec{H} \cdot \vec{H}$ & $\vec{H} \cdot \partial_{t} \vec{M}$ & $-\vec{H} \cdot \nabla \times \vec{P}$ & $\vec{K}_{b}$ \\
$\vec{D} \times \vec{B}$ & $\varepsilon_{0}^{-1} \vec{D} \cdot \vec{D}+\mu_{0}^{-1} \vec{B} \cdot \vec{B}$ & 0 & $-\vec{B} \cdot \nabla \times \vec{P}+\vec{D} \cdot \nabla \times \vec{M}$ & $\vec{K}_{P}, \vec{J}_{M}$ \\
\hline
\end{tabular}

\section{DISCUSSION}

Here we compare and contrast the residual terms, which neither appear in the form of an energy flux nor an energy density; they are summarized in table I. The Abraham $\vec{E} \times \vec{H}$ form has residuals that are purely dispersive, i.e. depend on the temporal derivatives of the medium responses via $\vec{P}$ and $\vec{M}$. In contrast, the Minkowski $\vec{D} \times \vec{B}$ form has residuals that are purely spatial i.e. depend on the curl of the medium responses. The lesser used forms $\vec{E} \times \vec{B}$ and $\vec{D} \times \vec{H}$ have mixed residuals - the $\vec{E} \times \vec{B}$ residuals conveniently match the form of the usual definition of the total current; whereas the $\vec{D} \times \vec{H}$ residuals match the less conventional picture of a material response described by bound monopoles.

Let us now consider the effects of these residual terms in all four cases, where we assume that $\vec{J}_{f}=0$.

For $\vec{E} \times \vec{H}$, the continuity eqn. (22) shows that the flux vs energy density balance is modified by two residual terms, i.e. the local dielectric excitation $\vec{E} \cdot \partial_{t} \vec{P}$, and local magnetic excitation $\vec{H} \cdot \partial_{t} \vec{M}$. Both of these are temporal, so if we choose to propagate forward in space, which is a common choice when considering propagation in optics (see e.g. [35, 39]), during each step forward in space, the fields $\vec{E}(t), \vec{H}(t)$ will be modified according to their time derivatives.

For $\vec{E} \times \vec{B}$, the continuity eqn. (29) shows that the flux vs energy density balance is modified by two residual terms, one temporal 
$\vec{E} \cdot \partial_{t} \vec{P}$, and one spatial $\vec{E} \cdot \nabla \times \vec{M}$; in concert they represent the local electrical work done on the charges comprising the current $\vec{J}$. Unlike in the optics $\vec{E} \times \vec{H}$ picture, here a propagation step forward in space would be complicated by the spatial term; likewise the alternate choice of a time propagation step would be complicated by the temporal term.

For $\vec{D} \times \vec{H}$, the continuity eqn. (33) shows that the flux vs energy density balance is modified by two residual terms, one spatial $\vec{H}$. $\nabla \times \vec{P}$, and one temporal $-\vec{H} \cdot \partial_{t} \vec{M} ;$ in concert they represent the local magnetic work done on the monople current $\vec{K}$. Just as for the $\vec{E} \times \vec{B}$ picture, this leads to complicated methodology for propagation of the fields.

For $\vec{D} \times \vec{B}$, the continuity eqn. (38) shows that the flux vs energy density balance is modified by two residual terms, both spatial: $-\vec{B}$. $\nabla \times \vec{P}$, and $\vec{D} \cdot \nabla \times \vec{M}$. Just as for the $\vec{E} \times \vec{H}$ picture, both residuals are of the same type (albiet spatial, not temporal), so that making the "causal" choice [44] to propagate in time gives us the simple case where the fields $\vec{D}(\vec{r}), \vec{B}(\vec{r})$ will be modified according to their spatial derivatives [45]. Despite this, propagation treatments that evolve the fields forward in time (e.g. FDTD [46], or [30, 47]) generally tend to persist with the use of $\vec{E}$ and $\vec{H}$.

\section{CONCLUSION}

We have summarized four distinct electromagnetic continuity equations, each being derived from (and being consistent with)
Maxwell's equations and the standard constitutive relations. Each handles the electric or magnetic response of the medium in a slightly different way, so the most appropriate form needs to be chosen according to the system under study.

Not only have we presented the different interpretations motivated by each of the Abraham $(\vec{E} \times \vec{H})$, Minkowski $(\vec{D} \times \vec{B})$, and standard electromagnetic ("electric current") $\vec{E} \times \vec{B}$ forms, but we also consider the alternative ("magnetic current") $\vec{D} \times \vec{H}$ form as well. Finally, we showed how a directional fields approach [31] could be used to generate a propagation equation for each flux vector. This propagation equation makes a relatively small number of assumptions, and so not only enables further interpretation of each flux and its interaction with the medium, but has potential applications in its own right - even in materials with a complex response [48], and for wideband or ultrafast optical pulses [49].

\section{Acknowledgments}

The authors acknowledge financial support from the Engineering and Physical Sciences Research Council (EP/E031463/1).
[1] M. Abraham, Rend. Circ. Mat. Palermo 28, 1 (1909).

[2] M. Abraham, Rend. Circ. Mat. Palermo 30, 33 (1910).

[3] H. Minkowski, Math. Ann. 68, 472 (1910), reprint of Minkowski, H., 1908, Nachr. Ges. Wiss. Goettingen, Math.- Phys. K1. 1908, 53.

[4] R. N. C. Pfeifer, T. A. Nieminen, N. R. Heckenberg, and H. Rubinsztein-Dunlop, Rev. Mod. Phys. 79, 1197 (2007), arXiv:0710.0461, doi:10.1103/RevModPhys.79.1197.

[5] J. H. Poynting, Proceedings of the Royal Society of London 38, 168 (1884), doi:10.1098/rspl.1884.0080.

[6] L. D. Landau and E. M. Lifshitz, Electrodynamics of Continuous Media (Pergamon, Oxford and New York, 1984).

[7] J. Slepian, J. Appl. Phys. 13, 512 (1942), doi:10.1063/1.1714903.

[8] C. S. Lai, Am. J. Phys. 49, 841 (1981), doi: $10.1119 / 1.12719$

[9] P. C. Peters, Am. J. Phys. 50, 1165 (1982), doi: 10.1119/1.13024.

[10] R. H. Romer, Am. J. Phys. 50, 1166 (1982), doi: 10.1119/1.12903

[11] D. H. Kobe, Am. J. Phys. 50, 1162 (1982), doi: $10.1119 / 1.12901$

[12] R. P. Feynman, R. B. Leighton, and M. Sands, The Feynman lectures on physics, vol. 2 (Addison-Wesley, 1964), 14 th printing.

[13] D. B. Melrose and R. C. McPhedran, Electromagnetic processes in dispersive media (Cambridge University Press, Cambridge, 1991), ISBN 0-521-41025-8.

[14] J. R. Reitz, F. J. Milford, and R. W. Christy, Foundations of electromagnetic theory (Addison-Wesley, 1980), 3rd ed.

[15] F. Richter, M. Florian, and K. Henneberger,

Europhys. Lett. 13, 117 (2008),

arXiv:0710.0515 doi:10.1088/0143-0807/13/3/003.

[16] M. W. McCall,

Metamaterials 2, 92 (2008),

doi:10.1016/j.metmat.2008.05.001.

[17] M. Hillery and L. D. Mlodinow, Phys. Rev. A 30, 1860 (1984), doi:10.1103/PhysRevA.30.1860.

[18] S. Datta, Eur. J. Phys. 5, 243 (1984), http: //www.iop.org/EJ/abstract/0143-0807/5/4/ $010 /$.

[19] C. J. Carpenter, IEE Proceedings - Science, Measurement and Technology 146, 73 (1999), doi:10.1049/ip-smt: 19990026.

[20] M. E. Crenshaw and N. Akozbek, Phys. Rev. E 73, 056613 (2006), https://doi.org/10.1103/PhysRevE.73.056613.

[21] I. Campos and J. L. Jimenez, Eur. J. Phys. 13, 117 (1992), 
http://www.iop.org/EJ/refs/0143-0807/13/3/003.

[22] Y. N. Obukhov and F. W. Hehl,

Phys. Lett. A 311, 277 (2003), doi : 10.1016/S0375-9601(03)00503-6.

[23] C. Raabe and D.-G. Welsch, Phys. Rev. A 71, 013814 (2005), see comment Pitaevskii-2006pra and reply Raabe-W-2006pra; also later comment Brevik-E-2008pra., doi:10.1103/PhysRevA.71.013814.

[24] V. A. Markel, Opt. Express 16, 19152 (2008), arXiv:0712.0605, doi:10.1364/OE.16.019152.

[25] L. P. Pitaevskii, Phys. Rev. A 73, 047801 (2006), doi:10.1103/PhysRevA.73.047801.

[26] C. Raabe and D.-G. Welsch, Phys. Rev. A 73, 047802 (2006), doi:10.1103/PhysRevA.73.047802.

[27] I. Brevik and S. A. Ellingsen, Phys. Rev. A 79, 027801 (2009), doi:10.1103/PhysRevA.79.027801.

[28] J. Z. Buchwald, From Maxwell to microphysics (University of Chicago, 1985), ISBN 0-226-07883-3.

[29] M. Kolesik, J. V. Moloney, and M. Mlejnek, Phys. Rev. Lett. 89, 283902 (2002), doi:10.1103/PhysRevLett.89.283902.

[30] M. Kolesik and J. V. Moloney, Phys. Rev. E 70, 036604 (2004), doi:10.1103/PhysRevE.70.036604.

[31] P. Kinsler, S. B. P. Radnor, and G. H. C. New, Phys. Rev. A 72, 063807 (2005), note that in this reference, the convolution symbol between the $\alpha_{c} \beta_{r}$ coefficents and the $G^{ \pm}$terms in square brackets in the frequency-domain propagation equations was inadvertently omitted, arXiv:physics/0611215v1, doi:10.1103/PhysRevE.75.066603.

[32] P. Kinsler (2006),

"Theory of directional pulse propagation: detailed calculations", arXiv:physics/0611216, http://arxiv.org/abs/physics/0611216.

[33] Y. Mizuta, M. Nagasawa, M. Ohtani, and M. Yamashita, Phys. Rev. A 72, 063802 (2005), doi:10.1103/PhysRevA.72.063802.

[34] A. Favaro, P. Kinsler, and M. W. McCall, Opt. Express 17, 15167 (2009), doi:10.1364/OE.17.015167.

[35] R. W. Boyd, Nonlinear Optics (Academic Press Inc., New York, 2008), 3rd ed., ISBN 978-0-12-369470-6, 1st ed. 1994, 2nd ed. 2003.

[36] T. Brabec and F. Krausz, Phys. Rev. Lett. 78, 3282 (1997), doi:10.1103/PhysRevLett.78.3282.
[37] M. Geissler, G. Tempea, A. Scrinzi, M. Schnürer, F. Krausz, and T. Brabec,

Phys. Rev. Lett. 83, 2930 (1999),

doi:10.1103/PhysRevLett.83.2930.

[38] P. Kinsler and G. H. C. New, Phys. Rev. A 67, 023813 (2003), arXiv:physics/0212016v1, doi: $10.1103 /$ PhysRevA.67.023813.

[39] P. Kinsler (2007),

"Pulse propagation methods in nonlinear optics", arXiv: 0707.0982 http://arxiv.org/abs/0707.0982.

[40] G. Genty, P. Kinsler, B. Kibler, and J. M. Dudley, Opt. Express 15, 5382 (2007), doi:10.1364/OE.15.005382.

[41] P. Kinsler, J. Opt. Soc. Am. B 24, 2363 (2007), the arXiv:0707.0986 version contains an additional appendix., arXiv:0707.0986v2, doi: $10.1364 /$ JOSAB .24 .002363

[42] P. Kinsler (2008),

"Transverse limits to the uni-directional pulse propagation approximation", arXiv: 0810.5701 http://arxiv.org/abs/0810.5701.

[43] P. Kinsler, Phys. Rev. A 81, 013819 (2010), arXiv:0810.5689 doi:10.1103/PhysRevA.81.013819.

[44] P. Kinsler,

Eur. J. Phys. 32, 1687 (2011), the arXiv version has additional appendices, arXiv: 1106.1792 doi : 10.1088/0143-0807/32/6/022.

[45] P. Kinsler (2015), "Temporally propagated optical pulses, and what they reveal about dispersion handling", arXiv: 1501.05569 http://arxiv.org/abs/1501.05569.

[46] K. S. Yee, IEEE Trans. Antennas Propagat. 14, 302 (1966), doi:10.1109/TAP.1966.1138693.

[47] M. Scalora and M. E. Crenshaw, Opt. Comm. 108, 191 (1994), doi : 10.1016/0030-4018(94)90647-5

[48] Various authors, Opt. Express 11, 639 (2003), "Focus Issue: Negative refraction and metamaterials", http://www .opticsinfobase.org/oe/issue.cfm? volume $=11 \&$ issue $=7$.

[49] T. Brabec and F. Krausz, Rev. Mod. Phys. 72, 545 (2000), doi:10.1103/RevModPhys .72.545. 